\title{
EDUCACIÓN Y CAMBIO CLIMÁTICO. UNA APROXIMACIÓN DESDE LA ESO
}

Education and Climate Change. An approach from ESO

\section{Manuela Caballero Guisado*; Artemio Baigorri Agoiz*; Mercedes} Pardo Buendía**

* Universidad de Extremadura; * Universidad Carlos III; manuelacaballero@unex.es; baigorri@unex.es; mpbuendi@polsoc.uc3m

\begin{tabular}{l} 
Historia editorial \\
\hline Recibido: 13-10-2017 \\
Primera revisión: 13-07-2020 \\
Aceptado: 16-04-2021 \\
Publicado: 1-07-2021 \\
\hline
\end{tabular}

Palabras clave

Cambio climático; Sistema Educativo; Ideología

\begin{abstract}
Resumen
La aceptación del cambio climático (CC) lleva implícito el reconocimiento de su origen antropogénico y la responsabilidad de las sociedades actuales para mitigarlo y reducirlo, una posición que tiene un sustrato ideológico que conecta con planteamientos políticos que defienden que el sistema educativo es la institución más adecuada para la Alfabetización Climática (AC) formal de las nuevas generaciones. En este artículo analizamos la situación de la AC en la etapa de la Educación Secundaria Obligatoria (ESO) en España desde la perspectiva de los administradores y educadores. Es una investigación exploratoria basada en técnicas cualitativas realizada en las Comunidades Autónomas de Madrid y Extremadura. Los resultados evidencian mínimas diferencias entre ambas Comunidades Autónomas en relación con el tratamiento que se hace del cambio climático en sus respectivos curriculum escolares a pesar de las diferencias ideológicas de sus gobiernos.
\end{abstract}

\section{Abstract}

Keywords

Climate Change; Educational Systems; Ideology
Acceptance of Climate Change (CC) implies the recognition of its anthropogenic origin and therefore the responsibility of current societies to mitigate and reduce it. This position has an ideological substrate that connects with political approaches that defend that the education system is the most appropriate institution for the Climate Literacy (CA) of the new generations. In this article we analyze the situation of CA in the Obligatory Secondary Education (ESO) stage in Spain from the perspective of administrators and teachers. It is an exploratory research based on qualitative methodology carried out in the Autonomous Communities of Madrid and Extremadura. The results show minimal differences between both $\mathrm{Au}$ tonomous Communities regarding the treatment of climate change in their respective school curricula despite the ideological differences of their governments.

Caballero Guisado, Manuela; Baigorri Agoiz, Artemio \& Pardo Buendía, Mercedes (2021). Educación y cambio climático. Una aproximación desde la ESO. Athenea Digital, 21(2), e2293. https://doi.org/10.5565/rev/athenea.2293

\section{Introducción}

El discurso generalmente aceptado por la inmensa mayoría de la comunidad científica se basa en asumir como causa principal del cambio climático (CC) las actividades antropogénicas, fruto de las emisiones de $\mathrm{CO}^{2}$ y de otros gases de efecto invernadero que aumentan significativamente desde el inicio de la época industrial. Así se constata con un $95 \%$ de certidumbre- por el Grupo Intergubernamental de Expertos sobre cambio climático (IPCC en sus siglas en inglés) en su último informe, publicado en 2014.

Desde finales del siglo XX, los sucesivos gobiernos españoles han firmado todos los protocolos internacionales sobre el clima, desde el primero (Río de Janeiro, 1992) al 
último (París, 2015), a pesar de partir de posiciones ideológicas muy distintas y con concepciones opuestas sobre $\mathrm{CC}^{1}$.

Un instrumento fundamental recomendado para concienciar sobre el reto que supone el CC para la sociedad en su conjunto es la educación, en concreto, la inclusión en los sistemas educativos formales e informales de lo que se conoce como "alfabetización climática". Atendiendo a los principios emanados de la US Global Change Research (2009), podemos definirla como la comprensión del impacto de los comportamientos individuales en el clima y de la influencia del clima en uno mismo y en la sociedad. Para Lesley Dupigny-Giroux (2010), la comprensión de esta interacción clima-sociedad no tiene que limitarse a la edad escolar, sino que debería extenderse a todos los ciudadanos y a lo largo de toda la vida.

En este contexto, nuestra pregunta de investigación fue: ¿cómo incorpora el sistema educativo español la alfabetización climática? El trabajo está diseñado como una investigación exploratoria, dada la escasa literatura sociológica ${ }^{2}$ existente sobre la presencia y desarrollo del concepto CC como componente del paquete socializador en el sistema educativo español. Y derivada de esta cuestión general aparecen otros interrogantes más específicos:

- Dado el nivel de descentralización del Estado español y la autonomía normativa de las distintas Comunidades Autónomas (en adelante CC. AA.) en materia educativa, es preciso, por un lado, conocer el tratamiento que la Administración central hace del concepto de $\mathrm{CC}$ en las asignaturas que regula; y por otro, comparar entre distintas CC. AA. para comprobar si existen diferencias en aquella parte del currículo que pueden legislar.

- Aceptando que el término CC aparece en el currículo establecido tanto a nivel nacional como autonómico, cabe preguntarse si su estudio es transversal al conjunto de las asignaturas, o específico de algunas de ellas.

Como en todo estudio exploratorio, cuando no se tienen antecedentes sobre el fenómeno estudiado o los existentes son imprecisos, nos vemos obligados a formular las hipótesis derivadas de las preguntas obtenidas al identificar el problema.

\footnotetext{
1 En el caso de España, el Think-Tank del Partido Popular (Fundación para el Análisis y Estudios Sociales/FAES) jugó un papel esencial en la difusión de las doctrinas negacionistas, ya bastante superadas.

2 Existen notables trabajos desde la Pedagogía, más específicamente desde la Educación Ambiental, entre los más recientes Araceli Serantes y Pablo Meira (2016), y otras propuestas interesantes no basadas en investigaciones empíricas (González, M. 2016). A partir de 2016, se ha producido en España, y en el mundo, una cierta eclosión de publicaciones sobre el tema, con monográficos de revistas como Documentación Social (n. ${ }^{\circ} 183$ ) o Papeles de Relaciones Ecosociales y Cambio Global (n. ${ }^{\circ}$ 138). En el momento de la revisión del artículo para su publicación hemos aprovechado para actualizar la información al respecto, así como para introducir algunos aspectos sugeridos por los revisores, cuyas aportaciones agradecemos.
} 
- Deducida de la normativa emanada del Ministerio de Educación, Cultura y Deporte al definir como troncales a las asignaturas que se imparten obligatoriamente en todas las CC. AA., afirmamos la escasa presencia del término CC en las asignaturas troncales de la ESO.

- Derivada de la capacidad normativa de las CC. AA. en materia educativa y por tanto de la posibilidad de introducir nuevos contenidos bajo la fórmula de asignaturas de Libre Configuración Autonómica (LCA), afirmamos que existen diferencias mínimas en el tratamiento y desarrollo que se hace del CC en las asignaturas de LCA en el currículo de la ESO en las comunidades objeto de estudio.

- Con la tercera de las hipótesis avanzamos que el concepto de CC en la ESO no se trabaja con carácter transversal en todas las asignaturas.

\section{Metodología}

La metodología se ha basado en la triangulación de herramientas cualitativas. El primer componente ha sido una amplia revisión bibliográfica sobre el tema que nos ocupa. En segundo lugar, se realizó un análisis documental de las leyes educativas en vigor tanto a nivel nacional como las de las Comunidades Autónomas de Madrid y Extremadura ${ }^{3}$. El proceso seguido ha consistido en localizar el término "cambio climático" en los contenidos, criterios de evaluación y estándares de aprendizaje evaluables de todas las asignaturas que componen el currículum de la ESO establecidas por la legislación vigente en el momento de la realización de este trabajo.

Finalmente, se realizaron entrevistas en profundidad, presenciales y semi-estructuradas, a los jefes de estudios de nueve Institutos de Enseñanza Secundaria (IES) del nivel de la ESO de ambas comunidades, entre enero y mayo de 2017. Inicialmente se optó por entrevistar a los jefes de estudio porque el desarrollo de sus funciones les permite tener una visión de conjunto de la programación anual, además de coordinar las actividades de carácter académico, extraescolares y complementarias del profesorado y del alumnado en relación con el proyecto educativo del centro. En algunas ocasiones los propios jefes de estudio nos remitieron al profesorado más implicado en estos temas, independientemente del área de conocimiento al que pertenecían.

En la selección de los centros educativos se buscó recoger en lo posible la diversidad del sistema educativo español, por lo que se atendieron criterios de titularidad

\footnotetext{
3 Aunque se había previsto inicialmente ampliar el análisis documental a los proyectos educativos de los centros seleccionados, se ha optado en aras de la eficiencia y en función de los medios disponibles por no incorporarlos, dadas las dificultades existentes para acceder a ellos en la generalidad de los centros educativos españoles.
} 
(pública/privada/concertada), socioeconómicos (rentas altas, medias y bajas), de innovación (pedagogías tradicionales y avanzadas) y territoriales (rural/urbano).

Para la investigación se ha seleccionado dos CC. AA. tradicionalmente polarizadas política y socio-económicamente, una selección consciente buscando de nuevo diversidad. Por un lado, la Comunidad de Madrid, la más rica del país, gobernada durante décadas por un partido conservador (Partido Popular, PP) que inicialmente participó de posiciones negacionistas del CC, y por otro, la Comunidad de Extremadura, en la cola en cuanto a nivel de riqueza, gobernada durante la mayor parte de su historia democrática por un partido progresista (Partido Socialista Obrero Español, PSOE) que desde el principio se sumó a la cuestión del CC (Heras, 2013).

La limitación de recursos disponibles para la realización del trabajo, así como la disponibilidad de los jefes de estudio para la realización de la entrevista, hace que los colegios finalmente seleccionados queden distribuidos de la siguiente forma: en Extremadura, seis colegios públicos y 1 colegio privado/concertado de carácter religioso; y en Madrid, un colegio privado/concertado de carácter religioso y otro colegio privado de carácter laico. El tratamiento de la información recabada en las entrevistas se realiza mediante el análisis comparativo de las respuestas de los informantes, que quedan anonimizados y caracterizados en la tabla 1.

\begin{tabular}{ccccc}
\hline Informante & Titularidad colegio & $\begin{array}{c}\text { Carácter } \\
\text { territorial }\end{array}$ & $\begin{array}{c}\text { Orientación reli- } \\
\text { giosa explícita }\end{array}$ & $\begin{array}{c}\text { Nivel socioeconómico } \\
\text { alumnado }\end{array}$ \\
\hline 1 & Público & Urbano & & Medio-alto \\
3 & Público & Rural & & Medio-bajo \\
4 & Público & Rural & Medio-bajo \\
5 & Concertado/Privado & Urbano & Católico & Medio-alto \\
6 & Privado & Urbano & Laico & Alto \\
7 & Concertado/Privado & Urbano & Católico & Alto \\
8 & Público & Urbano & & Medio \\
9 & Público & Urbano & & Medio \\
\hline
\end{tabular}

Tabla 1. Caracterización de informantes

De los niveles académicos optamos por analizar la ESO por ser una etapa de consolidación de conocimientos impartidos en primaria y de preparación para el Bachiller. Además, el alumnado está en la fase vital en la que la familia ha dejado de ser el principal agente socializador, interviniendo en la construcción de su personalidad otros 
agentes externos, como son el grupo de iguales; los medios de comunicación; , muy particularmente las nuevas tecnologías de la información y la comunicación - TIC-, que influyen poderosamente en la adquisición de valores y actitudes; y también los conocimientos y valores transmitidos en el sistema educativo sobre los que los jóvenes racionalizan en ese periodo sus creencias y valores (Taberner, 2008).

\section{Fundamentación teórica}

La base teórica de este trabajo descansa en tres componentes. En primer lugar, el componente que resulta del análisis de la estructura internacional creada para la lucha contra el CC. En segundo lugar, el de los retos educativos y de cambio social que plantea el CC. Por último, el de las políticas globales de respuesta frente al CC y, derivado de ello, el papel que se atribuye a la educación específica en CC.

\section{La evidencia y la lucha internacional contra el cambio climático}

Las primeras voces de alarma sobre la degradación ambiental se oyeron a lo largo de la década de los 50 y 60 del pasado siglo, tanto desde las Ciencias de la Naturaleza como desde las Ciencias Sociales (Bookchin, 1962; Carson, 1962; Commoner, 1971; Ehrlich, 1968; Meadows, 1972; Packard, 1961; Riesman, 1965). Centraron inicialmente sus denuncias en problemas evidentes de contaminación, desde entonces, la panoplia de problemas ambientales no ha hecho sino crecer abarcando temáticas nuevas a medida que se desarrollaban herramientas tecnológicas para la investigación medioambiental. Apareció ya desde los años 70 el CC como un tema específico (a veces bajo la expresión de Cambio Ambiental Global o de Calentamiento Global) que ha dado lugar a numerosas conferencias científicas y cumbres gubernamentales.

La organización internacional de lucha contra el CC se instrumentaliza con la Convención Marco de las Naciones Unidas sobre el cambio climático (CMNUCC), cuyo órgano político es la Conferencia de las Partes (COP), siendo el IPCC (Panel Intergubernamental de Expertos sobre cambio climático) el órgano científico. Son una constante en todos los informes del IPCC las recomendaciones para la mitigación y/o reducción de las emisiones, y la adaptación y/o resiliencia de las poblaciones a los posibles impactos y escenarios de riesgo. Y una de las fórmulas propuestas para ello son las políticas educativas dirigidas a la sensibilización e integración de estas cuestiones en los sistemas educativos formales e informales. 


\section{El negacionismo y/o escepticismo ante el cambio climático}

Una de las grandes limitaciones a la extensión de las políticas de lucha contra el CC es la existencia de posiciones negacionistas (en algunos casos autodefinidas como escépticas), que se articulan en torno a la Coalición Científica Internacional del Clima (ICSC). Esta organización, formada por científicos y expertos en energía, cuestiona tres elementos básicos comúnmente aceptados sobre el CC: que sea reciente e inusual en comparación con los registros históricos; que las emisiones humanas de dióxido de carbono y otros gases de efecto invernadero suponen un impacto peligroso para el clima; y que los modelos informáticos que emplea el IPCC sean indicadores fiables del clima futuro. Su enfoque principal es analizar las políticas de calentamiento global en función de sus implicaciones económicas, convirtiéndose en un poderoso think-tank con gran influencia en algunos de los líderes mundiales más poderosos.

Numerosos trabajos de investigación confirman la existencia de este movimiento de negación del CC e incluso documentan la evolución de su financiación y de su estructura organizativa (Boussalis y Coan, 2016; Brulle, 2014; Farrell, 2016a, 2016b). El sustrato teórico fundamental que soporta estas posiciones son las tesis anti-reflexividad (Dunlap et al., 2016; McCright y Dunlap, 2000; Rosa et al., 2014). En general, han sido defendidas por gobiernos conservadores, induciendo al escepticismo sobre el CC entre la población, especialmente entre aquellos ciudadanos más conservadores (McCright et al., 2016), sobre todo en los países anglosajones. En los países que componen la Unión Europea (UE) no ha tenido tanta influencia, aunque algunos gobiernos conservadores se hayan resistido a la aplicación de políticas de lucha y mitigación contra el CC.

No obstante, la opinión pública en la UE en relación con el CC no deja de presentar grandes contradicciones. Así lo recoge el informe elaborado por European Social Survey (2017) basado en los datos del Eurobarómetro 459 sobre CC y Energía, cuando, entre sus conclusiones, afirma que, aunque existe una abrumadora mayoría de la población europea que reconoce el origen antropogénico del CC (incluso en los países más escépticos), esa misma población no muestra gran preocupación por estos temas y sólo se sienten moderadamente responsables. Ese mismo informe sitúa a los españoles, tras los portugueses, como los ciudadanos europeos que indican estar más preocupados por el CC, "aunque es preciso recordar que los ciudadanos europeos, en conjunto, sitúan la pobreza, el hambre, la falta de acceso al agua potable o el terrorismo por delante del cambio climático como problemas más importantes para el mundo" (Lázaro et al., 2019, p. 5). 
En el caso de España, y según los datos aportados por el Real Instituto ElCano, "el cambio climático es de forma destacada el elemento que más preocupa a los españoles cuando se pregunta (...) por las amenazas a las que se enfrenta el mundo” (Lázaro et al., 2019, p. 14). En línea con esta preocupación, un año antes el CIS (2018), en el barómetro dedicado a CC, ya destacaba que el $88 \%$ de los encuestados creían que el CC obliga a un cambio en el funcionamiento de nuestras sociedades. Y ello a pesar de que precisamente España es uno de los escasos países de la UE en los que el negacionismo tuvo un fuerte peso político por el apoyo a dichas tesis desde los sectores más conservadores del Partido Popular (Baigorri y Caballero, 2018).

\section{Retos educativos y de cambio social que plantea el CC}

La alfabetización climática presenta dificultades específicas derivadas de la naturaleza y características del CC, que es un problema global que tiene capacidad para alterar la vida en el planeta y cuyo impacto pleno se manifestará en el medio y largo plazo; y que, aunque afecta a todos los países y poblaciones, lo hace de manera diferenciada, además de tratarse de un riesgo con muchas incertidumbres (Pachauri y Meyer, 2014), pero que necesariamente las sociedades están abocadas a abordar (Naciones Unidas, 2015).

Estas características desafían a los sistemas educativos formales e informales. Se trata de formar a los estudiantes en la Ciencia del Clima, pero, sobre todo, de capacitarles como ciudadanos que se enfrentan y enfrentarán aún más a medio plazo a un desafío mayúsculo. El CC deberá entenderse no sólo como una cuestión científica sino también, y principalmente, como un problema social complejo, caracterizado por la incertidumbre y la necesidad de toma de decisiones con un conocimiento incompleto del problema y de una gran complejidad científica. En esencia, la Alfabetización Climática se refiere "al aprendizaje de enfrentarnos a los riesgos, la incertidumbre y los cambios rápidos" (Stevenson et al., 2017, p. 1). En ese contexto, los sistemas escolares deben plantearse qué enseñar y cómo, así como la implicación y preparación de los estudiantes para ese futuro incierto. Es un gran desafío y no es una cuestión menor.

\section{El papel de la educación sobre el cambio climático}

Como se ha expuesto, las principales instituciones internacionales vinculadas al CC consideran la Educación como una herramienta fundamental para preparar a las generaciones actuales, pero sobre todo a las futuras, para enfrentar sus efectos. Solo por citar algunos ejemplos, en 2009 la Conferencia Mundial de la UNESCO sobre Educación para un Desarrollo Sostenible, celebrada en Bonn, abundó en esta idea desarrollando el 
programa Climate Change Learning Partnership y muchos países han empezado a desarrollar estrategias al respecto.

El Grupo de Trabajo sobre Objetivos, Audiencias y Estrategias en la Educación sobre el $\mathrm{CC}^{4}$, promovido por el Consejo Nacional de Investigación de los Estados Unidos, advirtió de cómo su país, a pesar de existir amplia información disponible, se enfrentaba a un vacío de conocimiento entre el público, tanto sobre los efectos como sobre los desafíos y, también, oportunidades del CC. Por ello planteaba la necesidad de mejorar la educación en el campo de las Ciencias de la Tierra, pero sobre todo sobre las dimensiones humanas y sociales del CC (Forrest y Feder, 2011). Por su parte, Raya Muttarak y Wolfgang Lutz (2014) revisan la literatura sobre el tema y concluyen que la educación es clave para desarrollar la capacidad adaptativa al cambio climático ${ }^{5}$.

Estas iniciativas conviene ponerlas en el contexto más amplio de la emergente y controvertida conceptualización de la "alfabetización climática", derivada de la alfabetización geocientífica, como parte de un movimiento más amplio de alfabetización científica.

Las personas que son alfabetas climáticas saben que la ciencia del clima puede informar nuestras decisiones para mejorar la calidad de vida. Tienen una comprensión básica del sistema climático, incluyendo los factores naturales y los causados por el ser humano que lo afectan (...) Son conscientes de la relación fundamental entre el clima y la vida humana y las muchas formas en las que el clima siempre ha jugado un papel en la salud humana. Tienen la capacidad de (...) utilizar esa información para apoyar sus decisiones. (DupignyGiroux, 2010, p. 204)

\section{Antonio García apunta a que:}

Su definición e incorporación en los currículos escolares ha pendulado entre dos enfoques: uno que se centra y enfatiza en la necesidad de orientar los procesos de enseñanza-aprendizaje en los contenidos científicos, y un segundo que apuesta por centrar los esfuerzos en las situaciones en las que la ciencia es clave en y para la vida cotidiana y la toma de decisiones. $(2017$, p. 1$)$

Todo ello sitúa el debate abierto para el desarrollo de la alfabetización y educación climática en el ámbito de la educación. Miguel Fuertes et al. (2020) han elaborado

\footnotetext{
${ }^{4}$ La administración Trump desmontó algunos de los organismos e incluso ordenado borrar páginas web relacionadas con el término "cambio climático" (Elcacho, 2017). Aunque perviven importantes plataformas gubernamentales como Global Change (http://www.globalchange.gov/)

5 Por supuesto, la propia Educación Ambiental, también respecto del CC, puede plantearse desde presupuestos ideológicos a veces antitéticos, como tempranamente evidenció Pablo Meira (2006), pero es un debate que va más allá de los objetivos de este trabajo.
} 
una propuesta de una herramienta basada en categorías para analizar la idoneidad de un currículum para alcanzar la competencia climática. Pues:

El cambio climático debería ser entendido no solo como una cuestión científica climática sino también, y principalmente, como un tema social complejo, de manera que por alfabetización y educación climática se trata sobre todo de aprender como sociedad a enfrentarnos a los riesgos, la incertidumbre y los cambios rápidos importantes. (Stevenson et al., 2017, p. 1)

En consecuencia, los países han establecido políticas orientadas en esta dirección. Por citar algunas iniciativas, cabe señalar la Alliance for Climate Education, que en los Estados Unidos ha formado desde 2008 a millones de niños y jóvenes, aportando recursos docentes para los centros educativos y los profesores (ACE, 2017). Por su parte, el Ministerio de Educación de Vietnam publicó tempranamente un manual educativo sobre el cambio climático, distribuido en todos los centros educativos y a través de Internet (Ministry of Education and Training, Live \& Learn and Plan in Vietnam, 2012).

Aunque algo más tardíamente que en los EE. UU., en Europa también se ha tomado conciencia de la importancia de la Educación para el CC. (Murphy et al., 2019). Nos centramos en Francia, Italia y Portugal, países latinos más cercanos culturalmente y, sobre todo, con más cercanía al tipo de problemas y riesgos del cambio climático comunes a España. En Italia se ha desarrollado en los últimos años varias redes de interés en torno al cambio climático (vinculadas a la Responsabilidad Social Corporativa, el Tercer Sector, o directamente de ámbito público, interministerial) que han promovido la AC como un paso fundamental y, en 2019, en el marco de la celebración de la Cumbre del Clima, el ministro de Educación informó de que la Educación para el CC iba a incorporarse de forma reglada en la Educación Secundaria. En Francia, diversas revistas de educación dedican monográficos a la educación en el Antropogeno, aunque sin citar explícitamente el cambio climático ${ }^{6}$. El Ministerio de Educación, siguiendo la estela de la iniciativa italiana en el año de la Cumbre del Clima, ha puesto en marcha en 2019 una serie de propuestas para el fortalecimiento de contenidos relacionados con el cambio climático, la biodiversidad y el desarrollo sostenible en los ciclos 1, 2, 3 y 4 de educación. Se pretende que todos los estudiantes, al finalizar su educación obligatoria, tengan el conocimiento y dominio esenciales de los conceptos fundamentales para comprender el calentamiento global, la destrucción acelerada de los ecosistemas naturales, el impacto de las actividades humanas en estos cambios y para medir los riesgos resultantes, para que puedan actuar y comprometerse en consecuencia (CSP, 2019). En Portugal, algunos actores contribuyen a la difusión de la alfabetización cien-

\footnotetext{
Así, el monográfico de Educaction et socialisation/ Les cahiers du CERFEE, dedicado a Environnements culturels et naturels : apprendre pour agir ensemble, editado por Centre de recherches sur les formes d'éducation et d'enseignement - section CNU 70 - Sciences de l'éducation, no recoge ningún artículo específico sobre AC.
} 
tífica y en el CC, aunque todavía no de forma reglada. Es el caso de la agencia Ciência Viva, creada en 1996 por el Ministerio de Ciencia y Tecnología como una unidad de apoyo para la Educación Científica y Tecnológica. Dependiente de la Agência de Inovação, se dedica básicamente a realizar estudios y programas en el ámbito de la promoción de la formación experimental en Ciencias y, en los últimos años, ha incorporado entre sus acciones la elaboración de materiales didácticos sobre el CC. Otro actor fundamental es también la Associação Portuguesa de Educação Ambiental (ASPEA), una ONG fundada en 1990 que ha introducido el CC entre sus objetivos de trabajo. Sin embargo, al contrario que en el planteamiento del Ministerio de Educación francés, buena parte de esas iniciativas se centran casi exclusivamente en los aspectos científico-naturales del problema, prestando muy poca atención a las dimensiones sociales del CC. Por otra parte, la literatura académica en portugués sobre Educación para el CC no es propiamente europea, sino de origen brasileño.

En el caso de España, y aunque inicialmente se abordó el tema con entusiasmo (Narbona, 2002), no se ha producido una estrategia unificada. De hecho, hasta muy recientemente no se ha considerado una "cuestión de Estado", sino que ha estado sujeta al debate político entre partidarios de las políticas de enfrentamiento del CC y negacionistas/escépticos. Desde principios de siglo, a través del Centro Nacional de Educación Ambiental (CNEAM) y la Oficina Española de cambio climático, se crean algunos repositorios de documentación educativa. En 2004 se promovió específicamente un Grupo de Trabajo sobre Comunicación, Educación y Participación frente al CC que ha realizado hasta 2016 trece seminarios monográficos. Sin embargo, desde 2011 sus implicaciones en el sistema educativo han sido escasas, cuando no nulas (González, 2012).

A falta de contenidos específicos en los currícula oficiales que lleguen a todo el alumnado, sí encontramos proyectos de educación ambiental con fuerte incidencia en los temas de CC, pero de alcance limitado al ser voluntaria la participación; algunos patrocinados por organismos públicos internacionales como la UNESCO (Climántica); otros nacionales como el propio CNEAM (agenda 21 escolar, hogares verdes, etc.) y otros puestos en marcha por grandes corporaciones privadas como IBERDROLA (Educaclima). Se trata de propuestas concretas que desarrollan determinadas competencias (Fuertes et al., 2020).

Algunos análisis son críticos con las políticas educativas desarrolladas. El proyecto RESCLIMA, centrado en la información y comunicación sobre CC, plantea que la alfabetización climática de la población es una condición insuficiente para cambiar actitudes y comportamiento en relación con el CC. Defienden la necesidad de conocer las representaciones sociales que tienen los distintos grupos sociales en base a las cuales 
construyen sus respuestas ante el CC. En uno de sus últimos trabajos analizan la presencia del CC en los libros de texto de secundaria, evidenciando la existencia "de un marco reduccionista y convencional, centrado exclusivamente en la dimensión ambiental, pero olvidando introducir la dimensión social” (Serantes y Meira, 2016, p. 164), limitación que, como veremos, se reproduce en la praxis educativa a través del proyecto educativo. A la misma conclusión llega el trabajo más exhaustivo de revisión de la literatura académica sobre estos temas realizado por Martha Monroe et al. (2017, p. 17, traducción propia) cuando afirman: "identificamos muy pocos programas educativos que intencionalmente aborden el cambio climático desde disciplinas sociales y científicas (multidisciplinarias, interdisciplinarias,o transdisciplinaria)”.

En España el debate político ha afectado hasta tal punto a la lucha contra el CC que durante casi una década no hemos dispuesto de elementos suficientes para evaluar las actitudes de la población. Desde 2007 hay evidencias de que el tema preocupa a la sociedad española, pues el $83 \%$ se mostraba ya entonces convencida de que se está produciendo un calentamiento global (Echevarren, 2012). Sin embargo, con la llegada del Partido Popular al gobierno en 2008, el principal organismo demoscópico de público (Centro de Investigaciones Sociológicas) dejó de incluir preguntas sobre CC en sus encuestas, existiendo únicamente algunos informes privados financiados por fundaciones empresariales (González y Meira, 2009; Baigorri y Caballero, 2018). Pero -aunque tenemos evidencias de que las crisis económicas reducen la preocupación de los ciudadanos por las cuestiones ambientales (Baigorri et al., 2012) - no está claro que eso haya incidido en una menor atención de la ciudadanía hacia al CC como se plantea en otros trabajos (Heras et al., 2016), pues una reciente encuesta comparativa internacional - realizada por la organización independiente Pew Research Center- sitúa a España en primera posición en cuanto a preocupación de la población: un $89 \%$ de los españoles se muestran preocupados por el CC, porcentaje por encima de otros problemas globales como el ISIS o la crisis económica (Poushter y Manevich, 2017). Asistimos, por tanto, al evaluar la escasa atención al CC, al efecto que posiciones ideológicas negacionistas han tenido en la acción pública. Y si esto es así, ha de impactar en las políticas educativas.

Por otra parte, las estrategias educativas vinculadas a los sistemas educativos formales son insuficientes para afrontar esta problemática, dadas las resistencias y obstáculos existentes (González y Meira, 2010). De hecho, Rick Flowers et al. (2009), muestran que la educación ambiental informal ha venido abriendo un extenso campo de acción, dada la diversidad de agentes socializadores. En Manuela Caballero (2020) se analizan, por ejemplo, las posibilidades que para el cambio de actitudes ambientales tiene el contacto intergeneracional. 
En los últimos dos años hemos asistido, también en España, a una cierta eclosión del tema a nivel institucional, en el marco de un cambio de tendencia gubernamental respecto a las cuestiones ambientales. La Cumbre del Clima de 2019 prevista en Chile, pero celebrada en Madrid, y la enorme atención mediática despertada por la adolescente Greta Thunberg y su expresión en el movimiento escolar de los Viernes por el Clima, alentaron una especial preocupación en todo el continente, produciéndose iniciativas institucionales que, a su vez, tuvieron un fuerte impacto mediático, como el anuncio del ministro de Educación de introducir la AC en Secundaria (Caballero y Baigorri, 2018). Incluso se ofertan cursos de iniciativa privada sobre cómo abordar el CC en el aula (Gallego, 2020) y algunas de las grandes multinacionales más señaladas como responsables de los impactos antropogénicos despliegan campañas de alfabetización climática, como la citada de IBERDROLA.

\section{Datos y resultados}

\section{Legislación nacional y autonómica}

La distribución de competencias educativas está regulada por la Ley Orgánica de Mejora de la Calidad Educativa (LOMCE). La norma establece en el art. 6 bis una distribución de competencias entre el Estado, las Comunidades Autónomas y los Centros Educativos. Distingue tres grupos de asignaturas: Troncales (T), Específicas (E) y de Libre Configuración Autonómica (LCA). A las Comunidades Autónomas se las faculta para completar el contenido de las troncales y establecer los contenidos tanto de las específicas como de las de LCA (art. 6 bis, 2. c). Respecto a los Centros, se dice que podrán completar los contenidos de los bloques de asignaturas Troncales, Específicas y de LCA (art. 6 bis, 2. c).

La modificación que la LOMCE realiza de los apartados 3 y 4 del artículo 120 de la LOE está dirigido a que las Administraciones Educativas potencien y promuevan la autonomía de los Centros Educativos. En la misma línea está la modificación que hace del artículo 121 de la LOE, tanto en el apartado 3 como en el nuevo apartado que se incorpora al texto.

Lógicamente, la posibilidad que tienen los gobiernos autonómicos y los centros educativos, aunque sea muy limitada, de introducir cambios en el currículo, permite (al menos desde el punto de vista normativo), una cierta diferenciación entre ellos, generando distintas "identidades" pedagógicas según las Autonomías y los centros. En cierto sentido podemos decir que el regulador facilita cierta diversidad educativa y, en 
teoría, aumenta las posibilidades que tienen los padres a la hora de elegir para sus hijos el producto educativo, es decir, el centro, más afín a sus valores.

Sin olvidar las preferencias ideológicas que los gobiernos puedan tener, es evidente que en el caso de España es complicado plantear una estrategia educativa sobre un tema concreto, al estar transferidas a las CC.AA. las competencias en materia de Educación. Es necesario descender al nivel autonómico para averiguar, atendiendo al objetivo de nuestra investigación, hasta qué punto el CC se ha integrado en el currículo, o al menos lo ha percolado directa o indirectamente.

\section{Sobre la autonomía de los centros educativos}

La Comunidad de Madrid, gobernada por el Partido Popular (PP) desde 1995, confiere autonomía a los centros educativos y les traslada facultades para configurar un proyecto docente propio en los aspectos curriculares. Así se recoge en el Decreto 13/2011 y en la Orden 2774/2011, de la Consejería de Educación y Empleo, por la que se desarrollan los Decretos de Autonomía de los Planes de Estudio en la Educación Primaria y en la Educación Secundaria Obligatoria, y se regula su implantación en los centros educativos de la Comunidad de Madrid. Sin ánimo de ser exhaustivos, en el desarrollo normativo posterior es necesario mencionar el Decreto 48/2015, que establece el currículo de la Educación Secundaria Obligatoria y, finalmente, la Orden 2160/2016 de la Consejería de Educación, Juventud y Deporte que aprueba las materias de L.C.A. en la Comunidad de Madrid.

Por su parte, la Ley 4/2011 de Educación de Extremadura es fruto del consenso político entre el gobierno de la Comunidad (PSOE) y el principal partido de la oposición (PP). En su exposición de motivos establece la autonomía de los centros educativos como pilar del sistema educativo extremeño, y -fundamentada en las posibilidades que otorga la LOE- el artículo 141 desarrolla los pormenores de la autonomía pedagógica.

En el desarrollo normativo posterior - y atendiendo al mandato de la propia Ley de Educación de Extremadura, mediante el Decreto 104/2013- se regula el funcionamiento de las Redes de Apoyo Social e Innovación Educativa (RASIE) de la Comunidad Autónoma de Extremadura y se crea la modalidad de Red de Escuelas de Investigación, Desarrollo Tecnológico e Innovación Educativa ${ }^{7}$. Tras la experiencia de gestión y evolución de los centros educativos en estas Redes se introducen algunos cambios que quedan recogidos en un nuevo Decreto, el 75/2015, por el que se regula el funciona-

\footnotetext{
En la actualidad existen siete redes con temáticas distintas, pero con una organización y funcionamiento muy similares. Una de estas Redes es la de Ecocentros.
} 
miento de las RASIE en los centros educativos sostenidos con fondos públicos de enseñanza no universitaria de la Comunidad Autónoma de Extremadura. Por último, tenemos que mencionar Decreto 98/2016, por el que se establecen la ordenación y el currículo de la Educación Secundaria Obligatoria y del Bachillerato para la Comunidad Autónoma de Extremadura.

A la vista de lo expuesto, el principio de autonomía de los centros establecido tanto por la LOE como por la LOMCE, ha sido transferido intacto desde las leyes centrales a las autonómicas, aunque, en virtud del desarrollo normativo descrito, parece que estamos ante dos modelos educativos con matices diferentes. Mientras que en la Comunidad de Madrid la iniciativa parte de cada uno de los centros, en la Comunidad de Extremadura la iniciativa la tiene el gobierno al proponer proyectos de Redes a los que los centros pueden adscribirse libremente.

\section{El término "cambio climático" en los currículos de la ESO}

Vistas las similitudes entre ambos modelos, a continuación analizamos si existen diferencias en el currículo de la ESO. Para ello utilizamos como medida el número de veces que el término "CC" aparece en los contenidos, los criterios de evaluación y los estándares de aprendizaje del currículo de secundaria. También se han tenido en cuenta en qué asignatura aparece y si la asignatura es troncal, específica o de LCA.

Tanto en la Comunidad de Madrid como en la Comunidad de Extremadura los currículos recogen las asignaturas troncales y específicas establecidas en las leyes educativas vigentes. (Tablas 2 y 3). En estas asignaturas, el término CC aparece en las siguientes:

- Valores Éticos. Asignatura de primer ciclo de la ESO. El término CC, aparece una sola vez como estándar de aprendizaje en el Bloque 6, denominado "Los valores éticos y su relación con la ciencia y la tecnología”.

4.3. Selecciona y contrasta información, en colaboración grupal, acerca de algunas de las amenazas que, para el medio ambiente y la vida, está teniendo la aplicación indiscriminada de la ciencia y la tecnología, tales como: la explotación descontrolada de los recursos naturales, la destrucción de hábitats, la contaminación química e industrial, la lluvia ácida, el cambio climático, la desertificación, etc. (Decreto 48/2015, p. 289)

- Ciencias Aplicadas a la Actividad Profesional. Asignatura de $4 .^{\circ}$ de la ESO. El término CC, aparece una sola vez como criterio de evaluación en el bloque 2 denominado Aplicaciones de la ciencia en la conservación del medio ambiente: 
2. Contrastar en qué consisten los distintos efectos medioambientales tales como la lluvia ácida, el efecto invernadero, la destrucción de la capa de ozono y el cambio climático. (Decreto 48/2015, p. 189)

- Cultura Científica. Asignatura de $4 .^{\circ}$ de la ESO. El término aparece dos veces: una en la introducción a la asignatura:

En la vida diaria estamos en continuo contacto con situaciones que nos afectan directamente, como las enfermedades, la manipulación y producción de alimentos o el cambio climático, situaciones que los ciudadanos del siglo XXI debemos ser capaces de entender (Decreto 48/2015, p. 201)

y otra como estándar de aprendizaje evaluable:

2.1. Reconoce los efectos del cambio climático, estableciendo sus causas. (Decreto $48 / 2015$, p. 204).

\begin{tabular}{|c|c|c|c|}
\hline & Primero ESO & Segundo ESO & Tercero ESO \\
\hline \multirow{8}{*}{$\begin{array}{l}\text { Asignaturas } \\
\text { troncales } \\
\text { (El horario lectivo míni- } \\
\text { mo de estas materias } \\
\text { ocupará, como mínimo, } \\
\text { el } 50 \% \text { del total del ho- } \\
\text { rario) }\end{array}$} & \multirow{3}{*}{ Biología y Geología } & \multirow{4}{*}{$\begin{array}{l}\text { Física y Química } \\
\text { Geografía e Historia }\end{array}$} & Biología y Geología \\
\hline & & & Física y Química \\
\hline & & & Geografía e Historia \\
\hline & \multirow{2}{*}{$\begin{array}{l}\text { Geografía e Historia } \\
\text { Lengua Castellana y } \\
\text { Literatura }\end{array}$} & & Lengua Castellana y Literatura \\
\hline & & $\begin{array}{l}\text { Lengua Castellana y Li- } \\
\text { teratura }\end{array}$ & Primera Lengua Extranjera \\
\hline & \multirow{3}{*}{$\begin{array}{l}\text { Matemáticas } \\
\text { Primera Lengua Ex- } \\
\text { tranjera }\end{array}$} & \multirow{3}{*}{$\begin{array}{l}\text { Matemáticas } \\
\text { Primera Lengua Extran- } \\
\text { jera }\end{array}$} & Materias de opción \\
\hline & & & $\begin{array}{l}\text { Matemáticas Orientadas a la En- } \\
\text { señanzas Académicas }\end{array}$ \\
\hline & & & $\begin{array}{l}\text { Matemáticas Orientadas a la En- } \\
\text { señanzas Aplicadas }\end{array}$ \\
\hline \multirow{3}{*}{$\begin{array}{l}\text { Asignaturas } \\
\text { Específicas } \\
\text { (en cada uno de los cur- } \\
\text { sos) }\end{array}$} & \multirow{3}{*}{\multicolumn{3}{|c|}{$\begin{array}{l}\text { Educación Física; Religión* y Valores Éticos* } \\
\text { Un mínimo de una y un máximo de cuatro de las siguientes asignaturas que } \\
\text { podrán ser diferentes en cada uno de los cursos: } \\
\text { Cultura Clásica, Iniciación a la Actividad Emprendedora y Empresarial, Música, Tec- } \\
\text { nología, Educación Plástica, Visual y Audiovisual, Segunda Lengua Extranjera, Reli- } \\
\text { gión }{ }^{* *} \text { y Valores Éticos }{ }^{* *}\end{array}$}} \\
\hline & & & \\
\hline & & & \\
\hline \multirow{2}{*}{$\begin{array}{l}\text { Asignaturas de } \\
\text { LCA }\end{array}$} & \multicolumn{3}{|c|}{$\begin{array}{l}\text { Lengua Cooficial y Literatura (recibirá un tratamiento análogo al de la materia de Len- } \\
\text { gua Castellana y Literatura) }\end{array}$} \\
\hline & \multicolumn{3}{|c|}{ Asignaturas específicas no cursadas o materias por determinar } \\
\hline
\end{tabular}

*Se cursarán en todos los cursos

** Si los padres, madres o tutores legales, o en su caso los estudiantes, no la han escogido anteriormente

En negrita, cursiva y subrayada las asignaturas donde aparece el término CC (la señalización es nuestra)

Tabla 2. Asignaturas troncales y específicas en el Primer Ciclo de la ESO 


\begin{tabular}{|c|c|c|}
\hline \multirow{9}{*}{$\begin{array}{l}\text { Asignaturas } \\
\text { Troncales } \\
\text { (El horario lectivo } \\
\text { mínimo de estas } \\
\text { materias ocupará, } \\
\text { como mínimo, el } \\
50 \% \text { del total del } \\
\text { horario) }\end{array}$} & $\begin{array}{c}\text { Enseñanzas Académicas } \\
\text { (hacia Bachillerato) }\end{array}$ & $\begin{array}{l}\text { Enseñanzas Aplicadas } \\
\text { (hacia la Formación Profesional) }\end{array}$ \\
\hline & \multicolumn{2}{|c|}{ Troncales Generales } \\
\hline & \multicolumn{2}{|l|}{$\begin{array}{l}\text { Geografía e Historia } \\
\text { Lengua Castellana y Literatura } \\
\text { Primera Lengua Extranjera }\end{array}$} \\
\hline & $\begin{array}{l}\text { Matemáticas orientadas a las ense- } \\
\text { ñanzas académicas }\end{array}$ & $\begin{array}{l}\text { Matemáticas orientadas a las enseñanzas aplica- } \\
\text { das }\end{array}$ \\
\hline & \multicolumn{2}{|c|}{ Troncales de cada opción } \\
\hline & A elegir dos entre las siguientes & A elegir dos entre las siguientes \\
\hline & Biología y Geología & Ciencias aplicadas a la actividad profesional \\
\hline & Economía & $\begin{array}{l}\text { Iniciación a la actividad emprendedora y empre- } \\
\text { sarial }\end{array}$ \\
\hline & Latín & Tecnología \\
\hline & \multicolumn{2}{|c|}{ Educación Física; Religión* y Valores Éticos ${ }^{*}$} \\
\hline $\begin{array}{l}\text { Asignaturas } \\
\text { Específicas }\end{array}$ & \multicolumn{2}{|c|}{$\begin{array}{l}\text { Un mínimo de una y un máximo de cuatro de las siguientes asignaturas que po- } \\
\text { drán ser diferentes en cada uno de los cursos: }\end{array}$} \\
\hline $\begin{array}{l}\text { (en cada uno de los } \\
\text { cursos) }\end{array}$ & \multicolumn{2}{|c|}{$\begin{array}{l}\text { Artes Escénicas y Danza, } \text { Cultura Científica, Cultura Clásica, Filosofía, Música, Tecno- } \\
\text { logía de la Información y la Comunicación, Segunda Lengua Extranjera, Educación Plás- } \\
\text { tica, Visual y Audiovisual, Una materia del bloque de asignaturas troncales no cursadas, } \\
\text { Religión }^{* *} \text { y Valores Éticos }\end{array}$} \\
\hline \multirow{3}{*}{$\begin{array}{l}\text { Asignaturas de } \\
\text { LCA }\end{array}$} & \multicolumn{2}{|c|}{$\begin{array}{l}\text { Lengua Cooficial y Literatura (recibirá un tratamiento análogo al de la materia de Lengua } \\
\text { Castellana y Literatura) }\end{array}$} \\
\hline & \multicolumn{2}{|c|}{ Asignaturas específicas no cursadas o materias por determinar } \\
\hline & \multicolumn{2}{|c|}{$\begin{array}{l}\text { Materias de ampliación de los contenidos de algunas de las materias de los bloques de } \\
\text { asignaturas troncales o específicas }\end{array}$} \\
\hline
\end{tabular}

*Se cursarán en todos los cursos

** Si los padres, madres o tutores legales, o en su caso los estudiantes, no la han escogido anteriormente

En negrita, cursiva y subrayada las asignaturas donde aparece el término CC (la señalización es nuestra)

Tabla 3. Asignaturas troncales y específicas en el Segundo Ciclo de la ESO

Estas son las coincidencias en ambos currículos. Para conocer sus diferencias hay que atender a las asignaturas de LCA. La Tabla 4 recoge las asignaturas de LCA aprobadas para la ESO por el gobierno de la Comunidad de Madrid mediante la Orden 2160/2016, de la Consejería de Educación, Juventud y Deporte. 


\begin{tabular}{lccc}
\hline \multicolumn{1}{c}{ Materia } & Curso ESO & $\begin{array}{c}\text { Departamento responsable } \\
\text { de su impartición }\end{array}$ & $\begin{array}{c}\text { Especialidad docente del } \\
\text { profesorado encargado de } \\
\text { impartirla }\end{array}$ \\
\hline $\begin{array}{l}\text { Comunicación Audiovisual Imagen } \\
\text { y Expresión }\end{array}$ & $3 .^{\circ}$ & Artes Plásticas & Dibujo \\
Botánica Aplicada & $3 .^{\circ}$ o $4 .^{\circ}$ & Ciencias Naturales & Biología y Geología \\
$\begin{array}{l}\text { Teatro } \\
3 .^{\circ} \text { o } 4 .^{\circ}\end{array}$ & Lengua Castellana y Literatura & Lengua Castellana y Literatura \\
$\begin{array}{l}\text { Dibujo Técnico y Diseño } \\
\text { Tecnología, Programación y Robóti- }\end{array}$ & $4 .^{\circ}$ & Artes Plásticas & Dibujo \\
ca. Proyectos Tecnológicos & $4 .^{\circ}$ & Tecnología & Tecnología \\
$\begin{array}{l}\text { Deportes } \\
\text { Ampliación de Matemáticas }\end{array}$ & $4 .^{\circ}$ & Educación Física y Deportiva & Educación Física \\
Ampliación de Física y Química & $4 .^{\circ}$ & Matemáticas & Matemáticas \\
\hline
\end{tabular}

Tabla 4. Asignaturas de LCA de la Comunidad de Madrid

El término de CC aparece una sola vez en la introducción de la asignatura de Ampliación de Física y Química para $4 .^{\circ}$ de la ESO, pero no forma parte de los contenidos. Resulta cuanto menos curioso que no se mencione el término en Botánica Aplicada, cuando en la introducción a la asignatura se explicita "la importancia que tienen para la especie humana los vegetales, en sus múltiples aspectos, desde su función en el mantenimiento del equilibrio medioambiental hasta la variedad de recursos que obtenemos de ellos" (BOCM, n. ${ }^{\circ}$ 155, p. 49).

En el caso de la Comunidad de Extremadura (Tabla 5) las asignaturas LCA son aprobadas mediante el Decreto 98/2016, por el que se establece la ordenación y el currículo de la Educación Secundaria Obligatoria y del Bachillerato.

\begin{tabular}{cc}
\hline Curso ESO & Asignatura \\
\hline $10^{\circ}$ & Segunda lengua Extranjera \\
$2 .^{\circ}$ & Segunda Lengua Extranjera, Educación para la Ciudadanía y Derechos Humanos \\
$3 .{ }^{\circ}$ & Segunda Lengua Extranjera, Cultura Clásica, Iniciación a la actividad emprendedora y empresarial \\
\hline
\end{tabular}

Tabla 5. Asignaturas de LCA de la Comunidad de Extremadura 
Dentro de estas asignaturas el término CC aparece una sola vez dentro de Educación para la Ciudadanía y Derechos Humanos y lo hace como estándar de aprendizaje evaluable en el bloque VI, denominado La ciudadanía en el siglo XXI.

La diferencia entre ambos currículos no es por tanto la cantidad de veces que aparece el término en sus contenidos, criterios y estándares de evaluación, que en ambas es escasísimo, sino que (por buscar matices) habría que centrarse en el tratamiento que se hace del concepto, en función de la asignatura en la que aparece referenciado. En la Comunidad de Madrid aparece en la introducción a la asignatura Física y Química con un carácter más técnico, aplicado al desarrollo económico y sus implicaciones "la competencia científica importante para comprender los problemas ambientales, médicos, económicos y de otro tipo a los que se enfrentan las sociedades modernas, que dependen enormemente del progreso tecnológico y científico” (DOCM, n. ${ }^{\circ}$ 155, p. 69).

La Comunidad de Extremadura, al ubicarlo dentro de la asignatura Educación para la Ciudadanía y Derechos Humanos, refuerza el carácter ético del concepto y las implicaciones individuales y colectivas en su abordaje.

Definir problemas medioambientales relacionados con la ciencia y la tecnología, así como en qué consiste el Protocolo de Kioto y la Cumbre del Clima de París de 2015. Propone actitudes cívicas relacionadas con el desarrollo sostenible y el respeto al medioambiente. Evalúa que combatir el cambio climático es un reto tanto individual como colectivo. (DOE, n. ${ }^{\circ} 129$, p. 7886)

Además de las asignaturas legalmente establecidas, en la Comunidad de Extremadura el programa de la Red Ecocentros trabaja específicamente los valores ambientales en aquellos centros que se adscriben a esta Red. Su filosofía, basada en el cambio social y la mejora ambiental, impregnaría el proyecto educativo de centro concretándose en el proyecto curricular. Sin embargo, y a pesar de que puede contribuir a la "ambientalización” general del alumnado, encontramos importantes limitaciones en el trabajo que en ellos se realiza sobre CC (Caballero y Baigorri, 2018).

En definitiva, es muy escasa, por no decir nula, la importancia que se le otorga en ambos currículos al CC. Así las cosas, las probabilidades de oír hablar en la ESO sobre CC oscila entre no haberlo oído en ninguno curso y haberlo oído en todos. Para ejemplificar el primer caso tenemos, tanto en Madrid como en Extremadura, a cualquier alumno de $4 .^{\circ}$ de la ESO que haya elegido el itinerario de Enseñanzas Académicas y que haya optado desde el primer curso por asistir a clases de Religión. Mientras que la mayor exposición al concepto la tienen los alumnos extremeños, siempre que elijan en los tres primeros cursos como asignatura optativa Valores Éticos; en 2. ${ }^{\circ}$ curso opten por Educación para la Ciudadanía; en $4 .^{\circ}$ cursen la modalidad de Enseñanzas Aplicadas 
y opten por las asignaturas de Ciencias Aplicadas a la Actividad Profesional y Cultura Científica.

\section{La implicación del profesorado: lo que se hace}

Aunque el análisis de los proyectos educativos no ha sido posible, al no estar accesibles en los centros educativos, el análisis de contenido de las entrevistas realizadas nos ha permitido conocer más en profundidad cómo trabajan los docentes el concepto de CC a pesar de su escasa presencia en el currículo.

En general los informantes coinciden en que la aproximación al concepto es voluntarista y depende del interés del profesorado por este tema. En la mayoría de las ocasiones se trabaja de forma muy periférica en las asignaturas de Ciencias Físicas y Biológicas, son escasas las veces que el concepto se menciona en asignaturas relacionadas con Ciencias Sociales y Humanas, y son muy pocos los centros en los que una amplia gama de asignaturas de distintas áreas de conocimiento relaciona sus contenidos con el CC. Este último caso lo encontramos en un colegio público cuando nos informan que "directamente se imparte en las asignaturas de Ciencias de la Naturaleza y Física y Química, pero también lo trabajamos en economía, inglés, filosofía y matemáticas" (informante 3, entrevista personal, marzo 2017). Al no tener un carácter troncal y trabajarlo de forma voluntaria, al profesorado le resulta difícil concretar el tiempo que invierten en ello, si bien algunos especifican "entre dos y tres horas a lo largo del curso" (informante 2, entrevista personal, febrero 2017).

En cuanto a los contenidos que se imparten de forma voluntaria sobre el CC, la mayoría del profesorado y en la mayoría de los centros priorizan su origen antropogénico y los impactos en los ecosistemas naturales, en asignaturas relacionadas con Ciencias Naturales y Biológicas.

Son los centros concertados y privados de carácter religioso, algunos centros públicos (con alumnado de extracción social media y media alta) y los que desarrollan pedagogías innovadoras con una fuerte conexión con la naturaleza, los que incluyen un trabajo más amplio del concepto incorporando las dimensiones sociales y humanas, la crítica de los modelos de producción y consumo, los mecanismos de resiliencia y adaptación a los cambios globales, las políticas internacionales de mitigación, entre otros asuntos. Todo ello con carácter transversal al resto de asignaturas. En referencia a estas cuestiones nos explican: "nosotros lo que hacemos, al trabajar el consumo responsable, es que vayan por lo menos pensándolo [las implicaciones personales y sociales que tiene] y que al final de su periodo en este colegio les quede algo" (informante 6, entrevista personal, enero 2017). 
Por tanto, el nivel de conocimiento sobre el CC del alumnado en la ESO es el resultado, por un lado, de la débil presencia del concepto en el currículo oficial, del itinerario curricular seleccionado y del centro elegido; y por otro, del desigual grado de interés, implicación y sensibilidad que el profesorado tenga con estos temas, pues en función de esto último se profundiza en mayor o menor medida dependiendo de la asignatura de que se trate.

Al margen de la ausencia del tema del $\mathrm{CC}$, en prácticamente la totalidad de centros seleccionados, existe algún tipo de programa más o menos extenso de educación ambiental en el que se priorizan los temas de ahorro en el consumo eléctrico y de agua, y la reutilización y reciclaje de materiales y equipos. Esta sensibilización ambiental se amplifica, en el caso de Extremadura, sólo en los colegios adscritos a la Red de Ecocentros. No obstante, estas actividades ni son sistemáticas, ni afectan a todo el alumnado. "Entre los años 2004-2008 hubo mucha implicación por parte de la administración, luego ha decaído mucho (...) No todo el alumnado participa en las actividades que se programan, solo aquellos cursos cuyos profesores se adscriben al proyecto" (informante 4, entrevista personal, abril 2017).

En esta situación general es preciso destacar algunas singularidades que sobrepasan al colectivo estudiado. En algunos centros públicos (extracción social de sus alumnos de clase media y media alta) tienen "programas internacionales de sensibilización sobre desarrollo sostenible para el alumnado de bachillerato" (informante 1, entrevista personal, enero 2017). En los centros concertados y privados de carácter religioso la educación ambiental-complementaria que recibe el alumnado se amplía con actividades autogestionadas por los propios estudiantes, como "equipos verdes, patrullas de limpieza o dedican un día a la semana a una acción ecológica. Por ejemplo, tener las luces menos tiempo encendidas" (informante 5, entrevista personal, mayo 2017). En la misma línea en otros centros: "En bachillerato se participa en el concurso Bgreen que son vídeos, anuncios de sensibilización ecológica" (informante 7, entrevista personal, febrero 2017). En otros colegios con pedagogías alternativas orientadas hacia una comprensión holista del ser humano y su relación con la naturaleza, "la sensibilización ecológica y el fomento de valores pro-ambientales forma parte de la personalidad del centro y contribuye a formar la conciencia y valores del alumnado" (informante 6, entrevista personal, enero 2017).

\section{Discusión y conclusiones}

La primera conclusión general que emerge de la investigación realizada es que el nivel de conocimiento del alumnado de la ESO en las comunidades estudiadas sobre CC es 
el resultado tanto de factores estructurales como de la agencia. La "path dependence" (Goldstone, 1998) y la desidia del sistema educativo español para incorporar de forma ágil conocimientos fundamentales para la vida en sociedad evidencia una clara dejación de la institución de sus funciones como agente de socialización. Esta desidia y/o reticencia al cambio funciona como barrera cultural a la innovación dentro de las instituciones educativas que reaccionan lentamente ante los desafíos del $\mathrm{CC}$, y se vincula en gran medida con el nivel de compromiso del gobierno de la nación con los acuerdos internacionales. Frente a la determinación estructural siempre nos encontramos con la agencia, en este caso, con el voluntarismo de parte del profesorado que sortea la falta de tiempo, los límites y oportunidades del currículo abordando por su cuenta la educación sobre el CC. Esta buena disposición por parte del profesorado es un capital social para la implementación de la alfabetización y educación climática una vez se elaboren políticas al respecto desde las Administraciones.

Sin embargo, aunque la transferencia entre conocimiento y comportamiento no siempre correlaciona de forma positiva, es evidente que la falta de alfabetización climática entre los escolares se presenta como una barrera en la resolución del problema. El conocimiento de las dimensiones físicas, sociales y humanas del $\mathrm{CC}$ al que se enfrentan las sociedades no puede quedar al arbitrio de la mayor o menor sensibilidad del profesorado con este tema.

Con la cautela con la que debe tomarse las conclusiones en un estudio exploratorio, podemos avanzar algunas que, no obstante, serían necesario confirmar en un estudio descriptivo más amplio. La mayor certeza de la investigación es sin duda la escasa presencia del concepto de CC en las asignaturas de la ESO ya sean troncales, específicas o de LCA. En este sentido, entendemos que existe desde todos los ámbitos de la Administración educativa un evidente desafecto con el reto que para nuestras sociedades supone el CC. Si de las instituciones educativas se espera que eduquen a los ciudadanos del futuro en el presente, resulta incomprensible la falta de previsión que se demuestra.

Este desafecto hacia el desafío del CC parece afectar también a las CC. AA. con independencia de la ideología de sus gobiernos; si bien es necesario matizar esta afirmación, porque sí hemos detectado ciertas diferencias que, aunque mínimas, son importantes desde el punto de vista cualitativo. Las diferencias encontradas en relación a la impartición de contenidos sobre CC dependen, sobre todo, de las actitudes y valores del propio profesorado, lo que lógicamente no permite esperar una acción coincidente.

Otra de las certezas es la práctica limitación del concepto de CC a los aspectos físicos, químicos y biológicos, quedando ausentes las dimensiones sociales y humanas, 
contraviniendo así las recomendaciones que los organismos internacionales de seguimiento del CC, de los que España forma parte, establecen.

\section{Financiación}

Este trabajo ha contado con la ayuda a grupos de investigación de la Junta de Extremadura y de los de fondos propios del Grupo de Investigación Análisis de la Realidad Social (ARS) de la Universidad de Extremadura.

\section{Referencias}

Alliance for Climate Education [ACE]. (2017). https://www.acespace.org/

Baigorri, Artemio \& Caballero, Manuela. (2018). El impacto del negacionismo en las políticas demoscópicas: el caso del cambio climático. Aposta. Revista de Ciencias Sociales, 77, 8-58. http://apostadigital.com/revistav3/hemeroteca/mcg2.pdf

Baigorri, Artemio; Caballero, Manuela \& Chaves, Mar. (2012). Cuando la crisis entra por la puerta, ¿la ecología sale por la ventana? En III Encuentro de Sociología y Medio Ambiente (SOCMAYS). Universidad de Alicante.

https://es.slideshare.net/artemiobaigorri/cuando-la-crisis-entra-por-la-puertala-ecologa-sale-por-la-ventana

Bookchin, Murray. (1962). Our Synthetic Environment. Knopf. http://dwardmac.pitzer.edu/Anarchist Archives/bookchin/syntheticenviron/ osetoc.html

Boussalis, Constantine \& Coan, Travis. G. (2016). Text-mining the signals of climate change doubt. Global Environmental Change, 36, 89-100. https://doi.org/10.1016/j.gloenvcha.2015.12.001

Brulle, Robert J. (2014). Institucionalizing delay: Fondation funding and the creation of U.S. climate change counter-movements organizations. Climate Change, 122(4), 681-694. https://doi.org/10.1007/s10584-013-1018-7

Caballero, Manuela. (2020). Quién apaga la luz. Generaciones y Medio Ambiente. Editamás/La Ciencia de la Sociedad

Caballero, Manuela \& Baigorri, Artemio. (2018). El cambio climático en la ESO. Una aproximación exploratoria en Extremadura. Revista de la Asociación de Sociología de la Educación. 11(1), 89-100. http://dx.doi.org/10.7203/RASE.11.1.10618

Carson, Rachel. (1962). Silent Spring. Houghton Mifflin.

CIS. (2018). Barómetro de noviembre. Estudio $n^{\circ} 3231$. http://datos.cis.es/pdf/Es3231mar A.pdf

Commoner, Barry (1971). The Closing circle. Knopf 
Conseil Supérieur des Programmes [CSP]. (2019). Note d'orientations et de propositions pour le renforcement des enseignements relatifs au changement climatique, à la biodiversité et au développement durable. Ministère de l'Éducation nationale, de la Jeunesse et des Sports.

https://www.education.gouv.fr/media/14153/download

Decreto 13/2011, de 24 de marzo, del Consejo de Gobierno, de autonomía de los planes de estudio de Educación Secundaria Obligatoria de la Comunidad de Madrid. BOCM. Núm. 89, 15 de abril 2011, pp. 57-58

Decreto 104/2013, de 18 de junio, por el que se regula el funcionamiento de las Redes de Apoyo Social e Innovación Educativa de la Comunidad Autónoma de Extremadura y se crea la modalidad de Red de Escuelas de Investigación, Desarrollo Tecnológico e Innovación Educativa. Diario Oficial de Extremadura. 21 de junio 2013, núm 121, pp. 14920-14947.

Decreto, el 75/2015, de 21 de abril, por el que se regula el funcionamiento de las Redes de Apoyo Social e Innovación Educativa en los centros educativos sostenidos con fondos públicos de enseñanza no universitaria de la Comunidad Autónoma de Extremadura. Diario Oficial de Extremadura. 27 de abril 2015, núm 79, pp.13557-13592

Decreto 48/2015, de 14 de mayo, del Consejo de Gobierno, por el que se establece para la Comunidad de Madrid el currículo de la Educación Secundaria Obligatoria. BOCM. Num 118. 20 de mayo de 2015, pp. 10-64

Decreto 98/2016, de 5 de julio por el que se establecen la ordenación y el currículo de la Educación Secundaria Obligatoria y del Bachillerato para la Comunidad Autónoma de Extremadura. Diario Oficial de Extremadura, 6 de julio de 2016, núm 129, pp 17347-18550

Dunlap, Riley E.; McCright, Aaron M. \& Yarosh, Jerrod H. (2016). The Political Divide on Climate Change: Partisan Polarization Widens in the U.S. Environment Science and Policy for Sustainable Development, 58(5), 4-23. https://doi.org/10.1080/00139157.2016.1208995

Dupigny-Giroux, Lesley A. (2010). Exploring the Challenges of Climate Science Literacy: Lessons from Students, Teachers and Lifelong Learners. Geographie Compass, 4(9), 1203-1217. https://doi.org/10.1111/j.1749-8198.2010.00368.x

Echevarren, José M. (2012). La percepción del cambio climático en España, en Artemio Baigorri (Ed.), Transiciones Ambientales. Ensayos de Sociología Ambiental (pp. 67-78). Amaru.

Elcacho, Joaquim (2017, 23 de enero). La Vanguardia. Esta es la web del cambio climático que Trump ha eliminado.

https://www.lavanguardia.com/natural/20170123/413624087974/web-casablanca-cambio-climatico-acceso-seguridad.html

Ehrlich, Paul. (1968). The population bom. Ballantine books.

European Social Science Survey. (2018). European attitudes to climate change and energy: topline results from round 8 of the European Social Survey. Autor. https://www.europeansocialsurvey.org/docs/findings/ESS 8 toplines issue 9 climatechange.pdf 
Farrell, Justin. (2016a). Corporate finance and ideological polarization on climate change. Proceedings of the National Academy of Sciences, 113(1), 92-97. https://doi.org/10.1073/pnas.1509433112

Farrell, Justin. (2016b). Network structure and influence of the climate change countermovements. Letters. Nature Climate Change, 6, 370-374. https://doi.org/10.1038/nclimate2875

Flowers, Ricks; Guevara, Roberto \& Whelan, James. (2009). Popular and Informal environmental education - The need for more research in an 'emerging' field of practice', Report, Zeitschrift fur Weiterbildungsforschung, 32(2), 36-50. http://www.die-bonn.de/doks/report/2009-umweltbildung-01.pdf

Forrest, Sherrie \& Feder Michael A. (2011). Climate Change Education: Goals, Audiences and Strategies. A Workshop Summary. National Academies Press.

Fuertes, Miguel Á.; Andrés, Santiago; Corrochano, Diego; Delgado, Laura; HerreroTeijón, Pablo; Ballegeer, Anne. M.; Lagos, Enzo F.; Fernández, Rubén y Ruiz, Camilo. (2020). Educación sobre el cambio climático: una propuesta de una herramienta basada en categorías para analizar la idoneidad de un currículum para alcanzar la competencia climática. Education in the Knowledge Society (EKS), 21. https://doi.org/10.14201/eks.22823

Gallego, José. (2020). Cómo abordar el cambio climático en el aula. Geoinnova. https://geoinnova.org/cursos/como-abordar-el-cambio-climatico-en-el-aula

García, Antonio. (2017, 26 de julio). Alfabetización climática: ¿Una solución real? RESCLIMA. http://www.resclima.info/node/192?language $=\mathrm{gl}$

Goldstone, Jack. (1998). Initial Conditions, General Laws, Path-Dependence, and Explanation in Historical Sociology. American fournal of Sociology, 104(3), 829-845. https://doi.org/10.1086/210088

González, Edgar \& Meira, Pablo. (2009). Educación, comunicación y cambio climático. Resistencias para la acción social responsable. Trayectorias, 11(29), 6-38.

González, Edgar \& Meira, Pablo. (2010). Climate Change Education and Communication: A Critical Perspective on Obstacles and Resistances. En Fumiyo Kagawa \& David Selby (Eds.), Education and climate change: living and learning in interesting times (pp. 13-34). Routledge.

González, Edgar. (2012). La representación social del cambio climático. Una revisión internacional. Revista Mexicana de Investigación Educativa, 17, 1035-1062.

González, María. (2016). Terminar la ESO sin conocer el cambio climático. Algunas reflexiones y herramientas para que esto no ocurra. PAPELES de Relaciones Ecosociales y Cambio Global, 136, 121-131.

Heras, Francisco. (2013). La negación del cambio climático en España: percepciones sociales y nuevos tratamientos mediáticos. En Rogelio Fernández-Reyes y Rosalba Mancinas (Eds.), Medios de comunicación y cambio climático (pp. 155170). Fénix Editora.

Heras, Francisco; Meira, Pablo \& Benayas, Javier. (2016). Un silencio ensordecedor. El declive del cambio climático como tema comunicativo en España 2008-2012. Redes.Com, 13, 31-55. 
Pachauri, Rajendra K. y Meyer, Leo (Eds.). (2014) Cambio climático 2014: Informe de sintesis. Contribución de los Grupos de trabajo I, II y III al Quinto Informe de Evaluación del Grupo Intergubernamental de Expertos sobre el cambio climático. IPCC.

Lázaro, Lara; González, Carmen \& Escribano, Gonzalo. (2019). Los españoles ante el cambio climático. Real Instituto Elcano.

http://www.realinstitutoelcano.org/wps/portal/rielcano es/contenido? WCM GLOBAL CONTEXT=/elcano/elcano es/zonas es/cambio-climatico/ encuesta-espanoles-ante-cambio-climatico-sep-2019

Ley 4/2011 de Educación de Extremadura. Diario Oficial de Extremadura. 9 marzo 2011, núm 47, pp. 5952-6035

Murphy, Cliona; Martínez, Gabriela; Aksela, Maija; Bergman, Gerd; Jones, Michael; Léna, Pierre \& Wilgenbus, David. (2020). A Snapshot of Climate Change Education Initiatives in Europe Initial findings and implications for future Climate Change Education. ALLEA.

McCright, Aaron; Charters, Meghan; Dentzman, Katerine \& Dietz Thomas (2016). Examining the effectiveness of climate change frames in the face of a climate change denial counter-frame. Topics in Cognitive Science, 8, 76-97. https://doi.org/10.1111/tops.12171

McCright, Aaron M. \& Dunlap, Riley E. (2000). Challenging global warming as a social being problem: An analysis of the counter-claims of conservative movement. Social Problems, 47, 499-522. https://doi.org/10.1525/sp.2000.47.4.03x0305s

Meadows Donella H. (1972). Los Límites del crecimiento: informe al Club de Roma sobre el predicamento de la humanidad. Fondo de Cultura Económica.

Meira, Pablo. (2006). El cambio climático y la educación ambiental neoliberal (que también existe). en Organismo Autónomo Parques Nacionales (Ed.), Reflexiones sobre educación ambiental II. Articulos publicados en la carpeta informativa del CENEAM 2000-2006 (pp. 55-65). Organismo Autónomo Parques Nacionales. Ministerio del Medio Ambiente

Ministry of Education and Training, Live \& Learn and Plan in Vietnam (2012). Teacher Manual on Climate Change Education. Compass Printing Company

Monroe, Martha C.; Plate, Richard, R; Oxarart, Annie; Bowers. Alison \& Chaves, Wilandia A. (2017): Identifying effective climate change education strategies: a systematic review of the research. Environmental Education Research, 25(6), 791-812, https://doi.org/10.1080/13504622.2017.1360842

Muttarak, Raya \& Lutz, Wolfgang (2014). Is education a key to reducing vulnerability to natural disasters and hence unavoidable climate change? Ecology and Society, 19(1), art. 42. https://doi.org/10.5751/ES-06476-190142

Naciones Unidas. (2015). Acuerdo de París. https://unfccc.int/files/essential background/convention/application/pdf/ spanish paris agreement.pdf

Narbona, Cristina. (2002). La prevención del cambio climático ¿límites tecnológicos o políticos? Observatorio Medioambiental, 5, 11-18. https://revistas.ucm.es/index.php/OBMD/article/view/OBMD0202110011A 
Orden 2774/2011, de 11 de julio, de la Consejería de Educación y Empleo, por la que se desarrollan los Decretos de Autonomía de los Planes de Estudio en la Educación Primaria y en la Educación Secundaria Obligatoria y se regula su implantación en los centros educativos de la Comunidad de Madrid. BOCM. Núm. 169, 19 de julio 2011, pp. 11-19

Orden 2160/2016, de 29 de junio, de la Consejería de Educación, Juventud y Deporte, por la que se aprueban materias de libre configuración autonómica en la Comunidad de Madrid. BOCM. Núm. 155. 1 de julio de 2016, pp. 41-69

Packard, Vance. (1961). Los artífices del derroche. Editorial Sudamericana.

Poushter, Jacob \& Manevich, Dorothy. (2017). Globally, People Point to ISIS and Climate Change as Leading Security Threats. Pew Research Center. https://www.pewresearch.org/global/2017/08/01/globally-people-point-toisis-and-climate-change-as-leading-security-threats/

Rosa, Eugene A.; Renn, Ortwin \& McCright, Aaron M. (2014). The Risk Society Revisited: Social Theory and Governance. Temple University Press.

Riesman, David. (1965). Abundancia ¿para qué? Fondo de Cultura Económica.

Stevenson, Robert; Nicholls, Jennifer \& Whitehouse, Hilary. (2017). What is climate change education? Curriculum Perspectives, 37(1), 67-71. https://doi.org/10.1007/s41297-017-0015-9

Serantes-Pazos, Araceli \& Meira, Pablo. (2016). El cambio climático en los libros de texto de la Educación Secundaria Obligatoria o una crónica de las voces ausentes. Documentación Social, 183, 153-170.

Taberner, José B. (2008). Sociología y educación. El sistema educativo en sociedades modernas. Funciones, cambios y conflictos. Tecnos.

US Global Change Research. (2009). Climate Literacy. The essential principles of Climate Science. https://downloads.globalchange.gov/Literacy/climate literacy highres englis $\underline{\text { h.pdf }}$

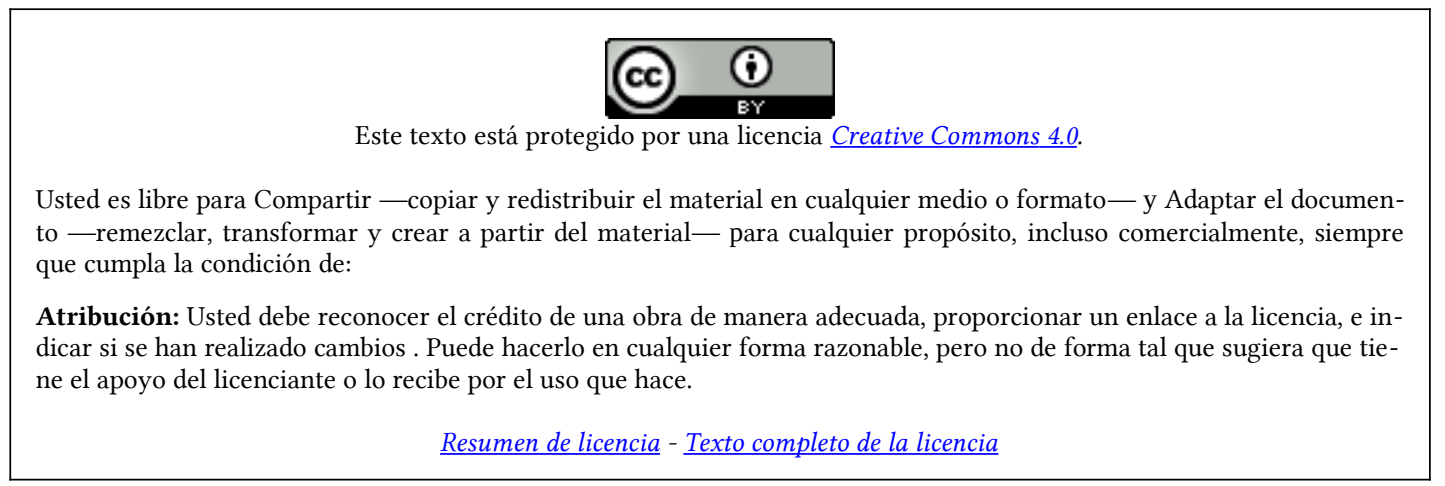

